\title{
DEVELOPMENT OF AN ONLINE LABORATORY: APPLICATION FOR THE CHARACTERIZATION OF NTC TEMPERATURE SENSOR
}

\author{
Ameur Ikhlef, Boubekeur Boukhezzar and Nora Mansouri \\ Laboratoire d'Automatique et de Robotique, Université Frères Mentouri, Constantine, Algérie \\ Route Ain ElBey, Université Frères Mentouri, Constantine 25000, Algérie
}

\begin{abstract}
This paper presents the development of practical experiences and their implementation on a remote Lab. Among the implemented experiences, we describe here the one that concerns the characterization of the NTC temperature sensor. For this experience, students collect data by varying gradually the temperature of a closed space and verify the effect of the variation of the temperature on the resistance of the sensor through a graphical interface. The measurement results are then saved automatically for further analysis. The remote control system was implemented using the LabVIEW environment whereas the platform that manages these i-Labs is based on the Interactive Shared Architecture ISA.
\end{abstract}

\section{KEYWORDS}

Remote Laboratories, Online Learning, Virtual Instrumentation, Sensors Characterization

\section{INTRODUCTION}

The development of Information and Communication Technologies (ICT), specially the internet, has widely contributed to introduce changes in many activities. Beyond the changes in the technical aspect, the different uses of these technologies have induced the emergence of and various efficient services. Today, ICT are present almost in all sectors including education and training. In many countries, the use of ICTs in education is well advanced and students are trained to use different forms of work offered by digital tools. In higher education, more and more universities propose to their students, in addition to traditional classroom teaching, remote activities. On the other hand, a growing number of universities suggest distance learning programs to people who wish to learn throughout their lives. The majority of these programs are based mainly on distance-learning courses and tutorials.

The development of distance laboratory works is a very active and attractive research topic, particularly in the scientific and technical fields where practice represents a very important part of the training (Aktan, Bohus, Crowl, \& Shor, 1996) (Leva \& Donida, 2008) (Wu \& Kuo, 2008) (Delong, et al., 2010) (Mujkanovic, Zutin, Schellander, Oberlercher, \& Vormaier, 2015).

Teaching laboratory works in classical way places the teacher in front with the following problems and constraints:

1. Massification: The increasing number of students does not allow to ensure laboratory works in suitable conditions. To do this, more equipment, more space, more time and more teachers are needed.

2. Equipment: Laboratory works require equipment that is often very expensive and bulky. It cannot be purchased in multiple copies for budget or space reasons.

3. Time: Time slots are often limited. That implies rotations and therefore restricts the number of laboratory works. It also implies that some students will have to do a laboratory work before addressing the topic in course

Therefore, remote laboratories are an interesting alternative to overcome all these problems while providing new features. New technologies bring onboard tools that facilitate access to machines that can be controlled, piloted and manipulated remotely. They allow the development of i-labs that offer to students and teachers new 
perspectives as well from a spatial and temporal point of view. I-labs experiments can be accessed remotely without having to move, saving time and reducing laboratory days of inactivity. Students can also redo the manipulations at suitable times. They just need to reserve their place beforehand.

The main advantage of these remote laboratories is therefore this possibility of being able to share the same experimental equipment between many users distributed in different geographical areas. Several educational teams around the world have already taken advantage of these technological advances to implement digital laboratories for distance learning.

In Frères Mentouri University of Constantine (UFMC), the development of physical remote laboratories (i-labs), is very active, particularly in the automatic control, electronics and electrical engineering areas. In this paper, we present the development of practical experiences and their implementation on the UFMC i-lab. For that, we organize it as follow: The architecture of UFMC i-Lab, the hardware and the software part are reported in section II. In section III, the objectives, technical content and procedure of the remote experiment are presented. In this section we present also the tests results of the remote experiment from remote-student side. Finally, conclusions and some perspectives are given.

\section{PRESENTATION OF THE UFMC I-LAB}

I-Labs are remote laboratories accessible through an Intranet or Internet network. They use web platforms to provide the users an interactive interface. This interface allows them to control hardware or drive real-time experiences via a simple web browser. To a remote access of these laboratories, the user needs to set up a platform that manages authentication problems, users flow, access to experiments, measurements visualization and recording and so on.

The UFMC i-lab platform was set up as a part of the Tempus E-science project: Maghreb network of distance laboratories (2012 - 2015). One of the project objectives is the setting up of three i-labs platforms in the Maghreb, including the UFMC i-Lab. At the end of the project, the UFMC i-Lab was in place with some e-Experiments developed by the UFMC team in the electronic and automatic fields (Hobar \& Semra, 2014) (Rebiai, Touidjen, \& Mouissat, 2014) (Latreche, Ziari, \& Mouissat, 2015) (Ikhlef, Kihel, Boukhezzar, Guerroudj, \& mansouri, 2015) (Ikhlef, Kihel, Boukhezzar, Mansouri, \& Hobar, 2016). These e-Experiments have been tested and evaluated by the different project partners.

\subsection{UFMC i-Lab Architecture}

The UFMC i-Lab is based on the ISA architecture that supports interactive labs (Harward, et al., 2008). This architecture allows the user to change the settings during the experiment. For the interactive labs, the simultaneous access of students to the same experience is impossible.

The UFMC i-Lab architecture is represented by the figure 1. As shown, the platform has a measurement server that manages experiments and a Web server that manages user access. Real-time remote visualization and control is performed using the LabVIEW Environment Web Server. In this case, the developed VI (Virtual Instrument) is available on the LAN or Internet through web browsers with an installed appropriate plug-in (JRE for applets based interfaces, runtime engine for a LabVIEW based remote experiments). The remote user only regains control of the experience after requesting and receiving acceptance from the server. As described above, this interactive interface gives students the opportunity to start and stop the experiment at their convenience. They can change the parameters of the experiment at any time from any terminal connected to the Intranet or Internet network. Moreover, it is also possible to store all data from experimental results. The UFMC i-Lab consists in 3 parts that complement each other: software, hardware and an experiments part:

\subsubsection{Software Part}

The UFMC i-Lab laboratory is based on the ISA architecture. This platform allows experiences deployment and sharing with different users. The platform is accessible through the university portal at http://ilab.umc.edu.dz/ilabservicebroker. The user logs to the platform via a username and a password. After the authentication phase, the user can communicate directly with the measurement server without having to go through the Web server each time. The first pass through the web server is required only to establish the connection with the experiment. 


\subsubsection{Hardware Part}

The hardware used in this experiment is represented by the figure 2 . The temperature chamber has a light heating resistor and three fans for cooling. The fan and resistor control boards have been designed for PWM control. In this case the cyclic ratio of the PWM signal will determine the rotation speed of the fans and the heating power of the resistor. The temperature inside the chamber is measured by an LM35 analog temperature sensor. To automate the temperature regulation, we have implemented two PID controllers; one for the fans and another for the heating resistor. The parameters of the controllers are previously identified by the Ziegler-Nichols method. It is also possible to implement self-tuning PID controllers.

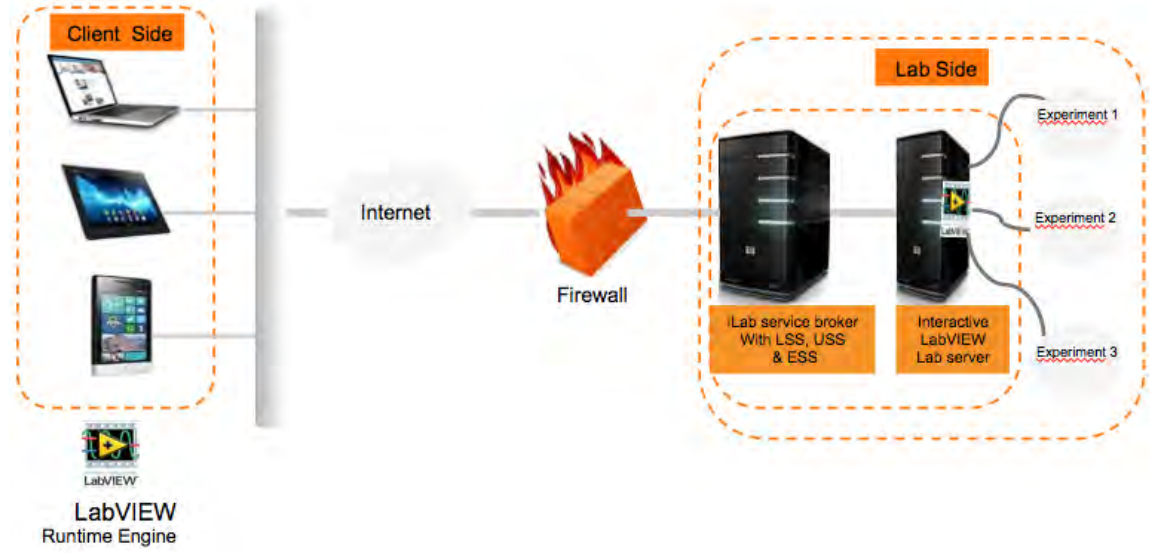

Figure 1. UFMC1 i-Lab architecture

The acquisition card is used to send the command signals to the heating resistor and the fans, and to retrieve the measurements delivered by the sensors.

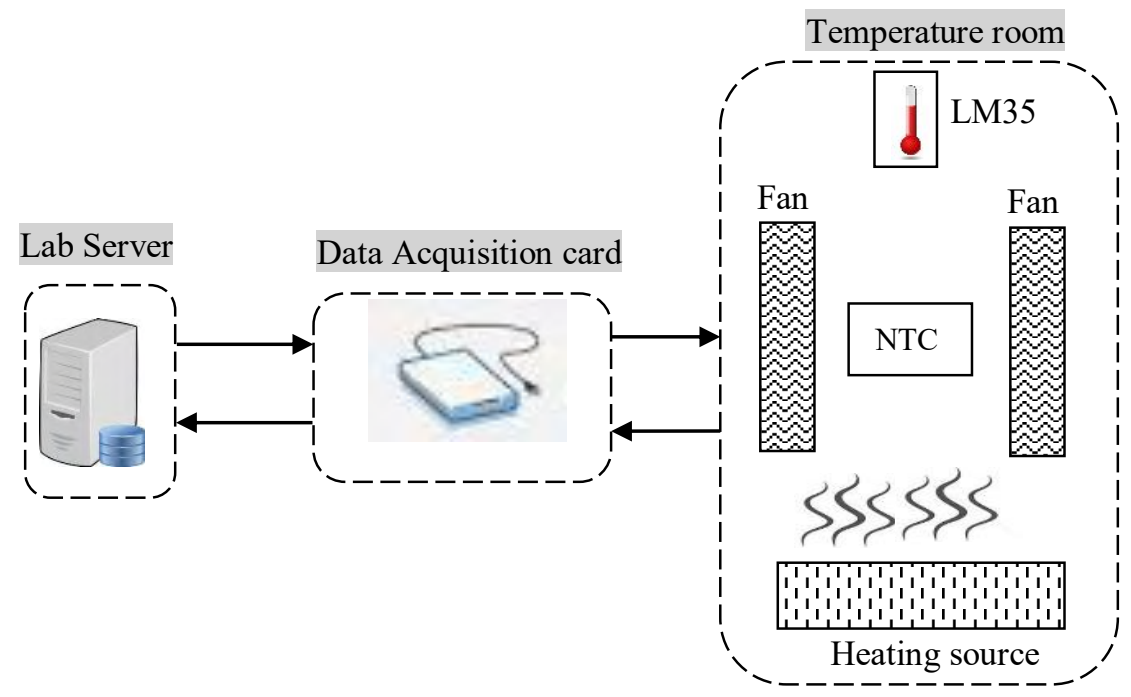

Figure 2. Hardware used in the NTC experiment

\section{NTC SENSOR CHARACTERIZATION EXPERIMENT}

Sensors are the basic elements of data acquisition systems. They are widely used in industrial systems, automated systems, and electronic instruments. A variation of the studied or measured physical phenomenon causes a variation on the physical and dimensional properties of the sensor. This variation is often expressed by a variation of voltage, current or impedance. In order to characterize the correspondence between the sensor 
output variation and the physical quantity to be measured, it is essential to establish a table or a calibration curve that represents the output of the sensor as a function of the physical quantity.

In this paper, the characterization of a temperature sensor (NTC thermistor) is considered. A thermistor is a temperature sensing element composed of a semiconductor material that exhibits a significant variation in resistance, proportional to a small change in temperature. Typically, a thermistor has negative temperature coefficients (NTC). It means that the resistance of the thermistor decreases as the temperature increases. The characterization of this thermistor consists in measuring the variation of the resistance value as a function of the temperature. In the classical method (hands on experiment), a NTC is placed in contact with a heating resistor powered by a voltage $V$. An Ohmmeter is connected to the terminals of the NTC and a thermometer is placed in contact with the heating resistor. The voltage $V$ is then slightly varied and the value of the NTC is recorded for each temperature.

For a remote characterization of the NTC thermistor, it is necessary to initially set up an automated system to control the manipulation. For this, a temperature control system is firstly realized. It is equipped with heat sources and a cooling device (fans) to stabilize the temperature to a desired value. Temperature regulation is provided by two PID controllers: one for the heat source and the other for the fans. This system can be used for other e-experiments such as regulation for example.

So, for the needs of the experience, we have executed the realization of the automated temperature room shown in Figure 3.
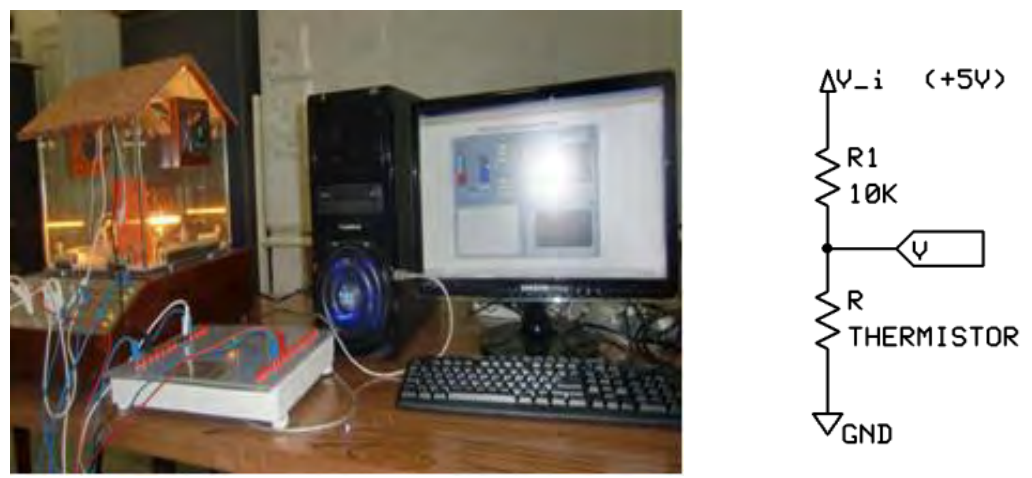

Figure 3. Temperature regulation room and the connection of NTC Sensor

The NTC is putted inside the temperature room and in order to simplify the sensor operation, It is placed in a voltage divider bridge as shown in Figure 3.

The resistor value is then given by:

$$
R=\frac{R_{1} \cdot V}{\left(V_{i}-V\right)}
$$

For the experience, the student increase gradually the temperature, the variation of the resistance is then continuously visualized as a function of the variation of the temperature. To drive the experiment, a graphical user interface (GUI) is developed using the LabVIEW environment. The graphical user interface is represented in Figure 4. From this interface, the student can choose a temperature value and the system automatically stabilizes the temperature of the room to the desired value. Once the system reaches the desired temperature, the student can use the Take Measurement button to capture the voltage across the NTC. The values will be automatically saved in a table. The heating-cooling button is used to select the heating or cooling cycle. The pull-down graphs display respectively the desired temperature steps and the voltage across the NTC. All measurement results are saved for later analysis. 


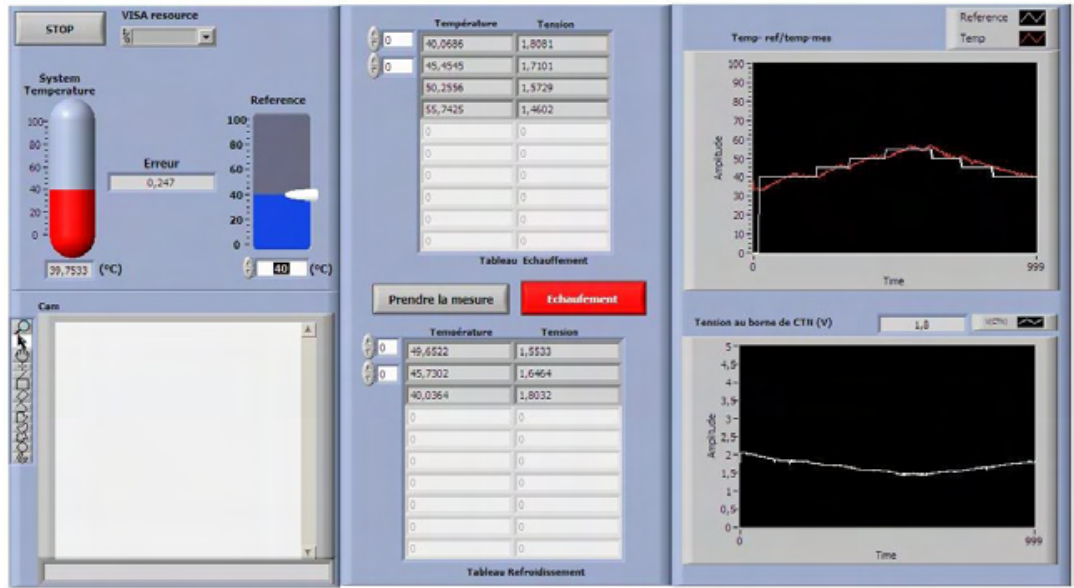

Figure 4. Graphical user interface

\section{CONCLUSION}

The NTC sensor characterization experiment exploits the UFMC web-based platform to give an interactive interface to the users. Thanks to the i-Lab, the experiment is available for all the subscribed students every day, 24 hours per day.

This learning method leads to save time and reduce inactivity days. For this remote experiment we noticed that the time allotted to the experiment decreased considerably compared to the classical method. So, it is possible now to share the experiment with the students of other specialties. Therefore, the use of these new technologies in the design of remote labs has given very satisfactory results whether in terms of pedagogy or logistics. As a perspective, it is also possible to use the same system to characterize other sensor types.

\section{REFERENCES}

Aktan, B., Bohus, C., Crowl, L., \& Shor, M. (1996). Distance learning applied to control engineering laboratories. IEEE Transactions on Education, 39(3), 320-326. doi:10.1109/13.538754

Delong, K., Harward, V. J., Bailey, P., Hardison, J., Kohse, G., \& Ostrocsky, Y. (2010). Three online neutron beam experiments based on the iLab Shared Architecture. IEEE EDUCON 2010 Conference. doi:10.1109/educon.2010.5492587

Harward, V., Alamo, J. D., Lerman, S., Bailey, P., Carpenter, J., Delong, K., . . Zych, D. (2008). The iLab Shared Architecture: A Web Services Infrastructure to Build Communities of Internet Accessible Laboratories. Proceedings of the IEEE, 96(6), 931-950. doi:10.1109/jproc.2008.921607

Hobar, F., \& Semra, L. (2014). Development and implementation of an e-course and a remote laboratory for analog electronics study. 2014 International Conference on Interactive Mobile Communication Technologies and Learning (IMCL2014). doi:10.1109/imctl.2014.7011109

Ikhlef, A., Kihel, M., Boukhezzar, B., Mansouri, N., \& Hobar, F. (2016). Online PID control of tank level system. 2016 IEEE Global Engineering Education Conference (EDUCON). doi:10.1109/educon.2016.7474566

Ikhlef, A., Kihel, M., Boukhezzar, B., Guerroudj, A., \& Mansouri, N. (2015). Online Temperature Control System. International Journal of Interactive Mobile Technologies, 9(2), 22-25. doi:10.3991/ijim.v9i2.4382

Latreche, S., Ziari, Z., \& Mouissat, S. (2015). UC1 Oscillator Remote Lab for Distant Electronics Education. International Journal of Interactive Mobile Technologies (iJIM), 9(2), 9. doi:10.3991/ijim.v9i2.4375

Leva, A., \& Donida, F. (2008). A remote laboratory on PID autotuning. IFAC Proceedings Volumes, 41(2), 8147-8152. doi:10.3182/20080706-5-kr-1001.01376

Mujkanovic, A., Zutin, D. G., Schellander, M., Oberlercher, G., \& Vormaier, M. (2015). Impact of students preferences on the design of online laboratories. 2015 IEEE Global Engineering Education Conference (EDUCON). doi:10.1109/educon.2015.7096067

Rebiai, S., Touidjen, N. E., \& Mouissat, S. (2014). Implementation of online optoelectronic devices course and remote experiments in UC1 iLab. 2014 International Conference on Interactive Mobile Communication Technologies and Learning (IMCL2014). doi:10.1109/imctl.2014.7011117

Wu, P., \& Kuo, C. (2008). The Design and Implementation of a Remote Automatic Control Laboratory: Using PID Control as an Example. Tamkang Journal of Science and Engineering, 11(2), 219-228. 